\section{Noninvasive assessment of hepatic steatosis using a pathologic reference standard: comparison of CT, MRI, and US-based techniques}

Jae Seok Bae ${ }^{1,2}$, Dong Ho Lee ${ }^{1,2}$, Kyung-Suk Suh ${ }^{3}$, Haeryoung Kim ${ }^{4}$, Kyung Bun Lee ${ }^{4}$, Jae Young Lee ${ }^{1,2,5}$, Joon Koo Han ${ }^{1,2,5}$

${ }^{1}$ Department of Radiology, Seoul National University Hospital, Seoul; ${ }^{2}$ Department of Radiology, Seoul National University College of Medicine, Seoul; Departments of ${ }^{3}$ Surgery and ${ }^{4}$ Pathology, Seoul National University Hospital, Seoul; ${ }^{5}$ Institute of Radiation Medicine, Seoul National University Medical Research Center, Seoul, Korea

Purpose: The present study compared the performance of computed tomography (CT), magnetic resonance imaging-derived proton density fat fraction (MRI-PDFF), controlled attenuation parameter (CAP), grayscale ultrasonography (US), and attenuation imaging (ATI) for the diagnosis of hepatic steatosis (HS).

Methods: In total, 120 prospectively recruited patients who underwent hepatic resection between June 2018 and June 2020 were retrospectively analyzed. CT, MRI-PDFF, CAP, grayscale US, and ATI were performed within 3 months before surgery. Diagnostic performance for HS $\geq 5 \%$ and $\mathrm{HS}>33 \%$ was compared using the area under the curve (AUC) of receiver operating characteristic curves. Histopathologic examinations served as the reference standard for the degree of HS.

Results: For detecting HS $\geq 5 \%$, MRI-PDFF (AUC, 0.946) significantly outperformed CT, CAP and grayscale US (AUC, 0.807, 0.829, and 0.761, respectively) ( $P<0.01$ for all). ATI (AUC, 0.892) was the second-best modality and significantly outperformed grayscale US ( $P=0.001)$. In pairwise comparisons, there were no significant differences between the AUC of ATI and the values of MRI-PDFF, $C T$, or $C A P(P=0.133, P=0.063$, and $P=0.150$, respectively). For detecting $H S>33 \%$, all the modalities provided good diagnostic performance without significant differences (AUC, 0.887-0.947; P>0.05 for all).

Conclusion: For detecting HS $\geq 5 \%$, MRI-PDFF was the best imaging modality, while ATI outperformed grayscale US. For detecting HS $>33 \%$, all five imaging tools demonstrated good diagnostic performance.

Keywords: Fatty liver; Nonalcoholic fatty liver disease; Diagnostic imaging

Key points: For detecting hepatic steatosis $\geq 5 \%$, magnetic resonance imaging-derived proton density fat fraction (MRI-PDFF) was the best imaging modality, while attenuation imaging (ATI) outperformed grayscale ultrasonography (US). For detecting hepatic steatosis $>33 \%$, all five imaging tools (computed tomography, MRI-PDFF, controlled attenuation parameter, grayscale US, and ATI) demonstrated good diagnostic performances.
ULTRA

SONO

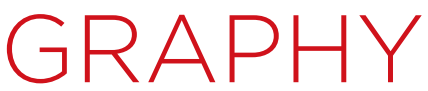

ORIGINAL ARTICLE

https://doi.org/10.14366/usg.21150 pISSN: 2288-5919 • elSSN: 2288-5943 Ultrasonography 2022;41:344-354

Received: July 18, 2021

Revised: September 29, 2021

Accepted: October 25, 2021

Correspondence to:

Dong Ho Lee, MD, Department of Radiology, Seoul National University Hospital, 101 Daehak-ro, Jongno-gu, Seoul 03080, Korea

Tel. +82-2-2072-3107

Fax. +82-2-743-6385

E-mail: dhlee.rad@gmail.com

This is an Open Access article distributed under the terms of the Creative Commons Attribution NonCommercial License (http://creativecommons.org/ licenses/by-nc/4.0/) which permits unrestricted noncommercial use, distribution, and reproduction in any medium, provided the original work is properly cited.

Copyright (C) 2022 Korean Society of Ultrasound in Medicine (KSUM)

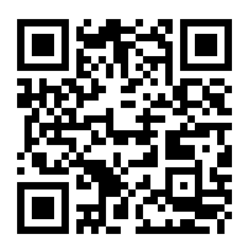

How to cite this article:

Bae JS, Lee DH, Suh KS, Kim H, Lee KB, Lee $J Y$, et al. Noninvasive assessment of hepatic steatosis using a pathologic reference standard: comparison of $\mathrm{CT}, \mathrm{MRI}$, and USbased techniques. Ultrasonography. 2022 Apr;41(2):344-354. 


\section{Introduction}

Hepatic steatosis (HS) is defined as the intracellular accumulation of triglycerides in more than $5 \%$ of hepatocytes [1]. HS has attracted interest because it is a signature feature of nonalcoholic fatty liver disease (NAFLD), which affects up to one-third of the global adult population and can progress to nonalcoholic steatohepatitis (NASH), fibrosis, or liver cirrhosis [2]. Ultimately, NAFLD can lead to endstage liver disease, which necessitates liver transplantation, or to the development of hepatocellular carcinoma [3]. Indeed, NASH is currently the leading cause of liver transplantation for women in the United States [3]. NAFLD has also been reported to be associated with metabolic syndrome and cardiovascular disease [4]. However, NAFLD can be treated in its early stages by lifestyle modifications such as weight loss, and currently, there are a few ongoing clinical trials on the treatment of NAFLD $[4,5]$. Therefore, it is of substantial clinical importance to detect and quantify HS and to monitor the changes in $\mathrm{HS}$.

Although liver biopsy has been the reference standard for the evaluation of HS [6], its invasiveness renders it unsuitable for use in clinical practice and research. Therefore, various tools, such as grayscale ultrasonography (US), computed tomography (CT), magnetic resonance imaging-derived proton density fat fraction (MRI-PDFF), and the controlled attenuation parameter (CAP) from transient elastography (TE), have been used for the noninvasive evaluation of HS $[7,8]$. Among them, MRI-PDFF is the most accurate method for HS detection and it has been used as a noninvasive reference standard for the evaluation of HS in recent clinical trials [7]. In contrast, quantitative US techniques, such as attenuation imaging (ATI), based on two-dimensional US images have been recently introduced and have demonstrated promising results for the assessment of HS [9-17]. These quantitative US techniques have the advantages of being more accessible and less expensive than CT or MRI-PDFF [8]. Although a few studies have compared the diagnostic performance of US, CT, and MRI for the assessment of HS [18-20], no study has compared the diagnostic performances of all available imaging tools, including CAP or other quantitative US techniques (e.g., ATI), using robust reference standards. Considering the emerging role of quantitative US techniques, including CAP, for the management of patients with NAFLD [10,21], information on the relative diagnostic performance of imaging tools would be valuable for selecting optimal imaging examinations to evaluate patients with HS. Therefore, the aim of this study was to compare the diagnostic performance of CT, MRI-PDFF, CAP, grayscale US, and ATI for the evaluation of HS in patients using pathologic reference standards.

\section{Materials and Methods}

\section{Compliance with Ethical Standards}

This retrospective study was approved by the institutional review board (IRB No. H-2007-027-1139) with a waiver of informed consent.

\section{Patients}

A retrospective analysis was conducted of 134 prospectively enrolled consecutive patients who underwent liver resection for suspected malignancy at Seoul National University Hospital between June 2018 and June 2020. The study coordinator (J.S.B., a board-certified radiologist with 8 years of experience in abdominal imaging) reviewed their electronic medical records and imaging examinations to identify patients who met the following inclusion criteria: (1) patients who underwent liver resection for suspected malignancy and (2) patients $\geq 19$ years old. Among the 134 patients, 14 patients were excluded for the following reasons: MRI-PDFF was not performed in patients who were referred from outside hospitals $(n=11)$; the interval between any of the imaging examinations and liver resection was $>3$ months $(n=1)$; heterogeneous fatty infiltration in the liver was visible on MRI-PDFF (described below) $(n=1)$; and splenectomy was performed $(n=1)$ (Fig. 1). Patients who underwent splenectomy were excluded because splenic attenuation was required for the assessment of HS on CT. Finally, this study included 120 patients who underwent CT, MRI-PDFF, TE, grayscale US, and ATI within 3 months before surgery and whose baseline characteristics are presented in Table 1. Of note, the final 120 patients were assessed in a previous study [22]. However, the previous study assessed the role of shear wave elastography in predicting post-hepatectomy complications, whereas this study compared the diagnostic performance of imaging modalities in

134 Consecutive adult patients who underwent hepatic resection for suspected malignancy between June 2018 and June 2020

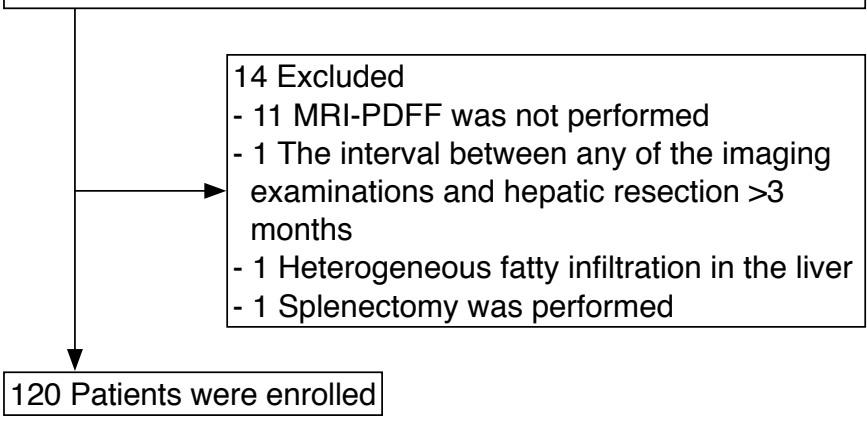

Fig. 1. Study flow diagram. MRI-PDFF, magnetic resonance imaging-derived proton density fat fraction. 
Table 1. Characteristics of the study population

\begin{tabular}{|c|c|}
\hline Characteristic & Value $(n=120)$ \\
\hline Age (year) & $60.7 \pm 10.5$ \\
\hline Male sex & $81(67.5)$ \\
\hline BMI $\left(\mathrm{kg} / \mathrm{m}^{2}\right)$ & $24.0(22.5-26.5)$ \\
\hline \multicolumn{2}{|l|}{ Etiology of chronic liver disease } \\
\hline HBV & $74(61.7)$ \\
\hline NAFLD & $8(6.6)$ \\
\hline $\mathrm{HCV}$ & $6(5.0)$ \\
\hline Alcohol & $4(3.3)$ \\
\hline HBV and alcohol & $2(1.7)$ \\
\hline Unknown & $3(2.5)$ \\
\hline No chronic liver disease & $23(19.2)$ \\
\hline \multicolumn{2}{|l|}{ Laboratory results } \\
\hline AST & $27(21-34)$ \\
\hline ALT & $24(18-37)$ \\
\hline Albumin (g/dL) & $4.2 \pm 0.4$ \\
\hline Bilirubin (mg/dL) & $0.6(0.5-0.8)$ \\
\hline PT-INR & $0.99(0.96-1.05)$ \\
\hline Platelet count $\left(\times 10^{3} / \mathrm{mm}^{3}\right)$ & $189 \pm 58$ \\
\hline \multicolumn{2}{|l|}{ Liver cirrhosis on pathology } \\
\hline Yes & $43(35.8)$ \\
\hline \multicolumn{2}{|l|}{ Degree of HS on pathology (\%) } \\
\hline$<5$ & $54(45.0)$ \\
\hline$\geq 5-33$ & $50(41.7)$ \\
\hline$\geq 5-10$ & $30(25.0)$ \\
\hline$\geq 10-33$ & $20(16.7)$ \\
\hline$>33-66$ & $14(11.7)$ \\
\hline$>66$ & $2(1.7)$ \\
\hline
\end{tabular}

Values are presented as mean $\pm \mathrm{SD}$, number (\%), or median (interquartile range). $B M I$, body mass index; HBV, hepatitis B virus; NAFLD, nonalcoholic fatty liver disease; HCV, hepatitis C virus; AST, aspartate transaminase; ALT, alanine transaminase; PTINR, prothrombin time-international normalized ratio; HS, hepatic steatosis.

detecting $\mathrm{HS}$.

\section{CT Examination}

Unenhanced CT scans were used for the assessment of HS by using the previously established CT index, which was calculated as hepatic attenuation minus splenic attenuation [23]. The study coordinator (J.S.B.) performed measurements of hepatic and splenic attenuation by placing regions of interest (ROIs) on the $\mathrm{CT}$ images 1 month after patient inclusion to avoid recall bias (Fig. 2A). The details of the CT protocol and ROI measurements are described in the Supplementary Data 1.

\section{MRI Examinations Including MRI-PDFF}

At the authors' institution, multiecho Dixon MRI-PDFF is incorporated into routine liver MRI examinations. PDFF is calculated as the ratio of MRI-visible fat protons to the sum of MRI-visible fat and bulk (free) water protons [24]. On the PDFF map automatically generated by each vendor's algorithm, the radiologist (J.S.B.) performed measurements by manually placing circular ROls in each of the nine Couinaud segments of the liver, and the average PDFF value was used [25] (Fig. 2B). The heterogeneity of fatty infiltration of hepatic parenchyma on the PDFF map was also assessed, and patients with suspected uneven fatty liver were excluded because it could be difficult to determine the degree of HS. The details of MRIPDFF are provided in the Supplementary Data 1.

\section{CAP Examinations}

At the authors' institution, TE is routinely performed in surgical candidates for hepatic resection to predict postoperative outcomes [26]. The CAP was measured by using a TE device (Fibroscan, Echosens, Paris, France) (Fig. 2C). TE examinations were performed until a minimum of 10 valid liver stiffness values were obtained, and CAP was calculated only when the liver stiffness measurements were confirmed to be valid. The median CAP value $(\mathrm{dB} / \mathrm{m})$ obtained from reliable TE examinations was used for analysis. The details are described in the Supplementary Data 1.

\section{Grayscale US Examinations}

The grayscale US examinations were performed by one of two radiologists (D.H.L. and J.S.B. with 15 and 8 years of experience in abdominal imaging, respectively) who were informed that the patients would undergo hepatic resection but were blinded to other clinical information. The patients fasted for at least 6 hours, and a US machine (Aplio i900, Canon Medical Systems, Tokyo, Japan) equipped with a 1-8-MHz convex probe was used (Fig. 2D). The radiologist (J.S.B.) qualitatively graded HS on grayscale US according to the following criteria: mild steatosis for increased hepatorenal contrast due to bright hepatic parenchymal echogenicity; moderate steatosis for vessel blurring; and severe steatosis for nonvisualization of the diaphragm due to posterior beam attenuation [27]. The details are described in the Supplementary Data 1.

\section{ATI Examinations}

Immediately after the grayscale US examination, ATI examinations were performed. While securing an adequate sonic window through an intercostal window for the right liver lobe, the ATI mode was activated. A color-coded sampling box was positioned in the liver parenchyma. In the sampling box, structures other than the liver parenchyma such as large vessels were automatically excluded by 


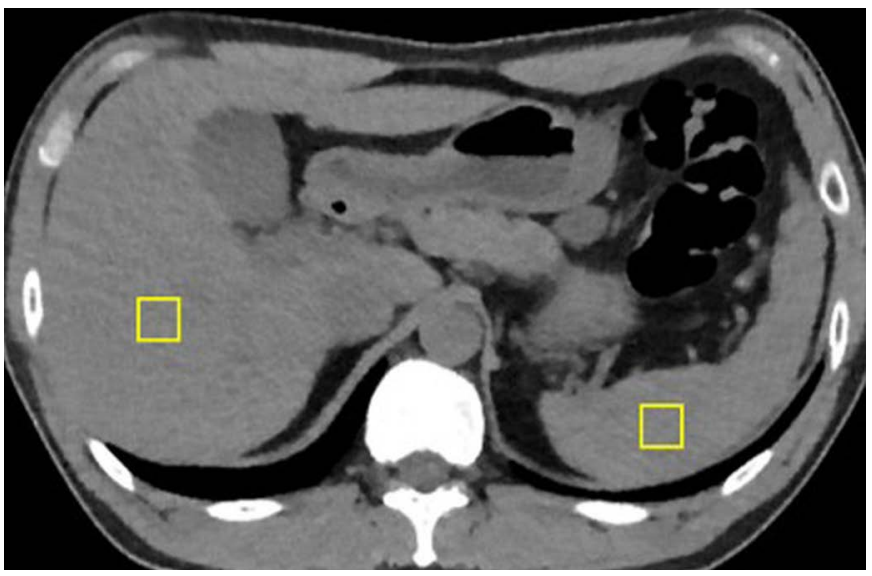

A

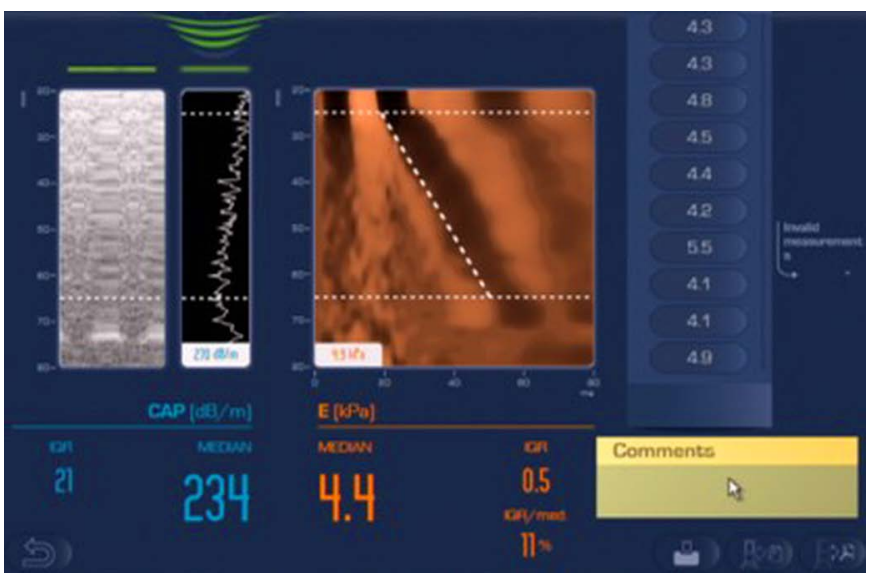

C

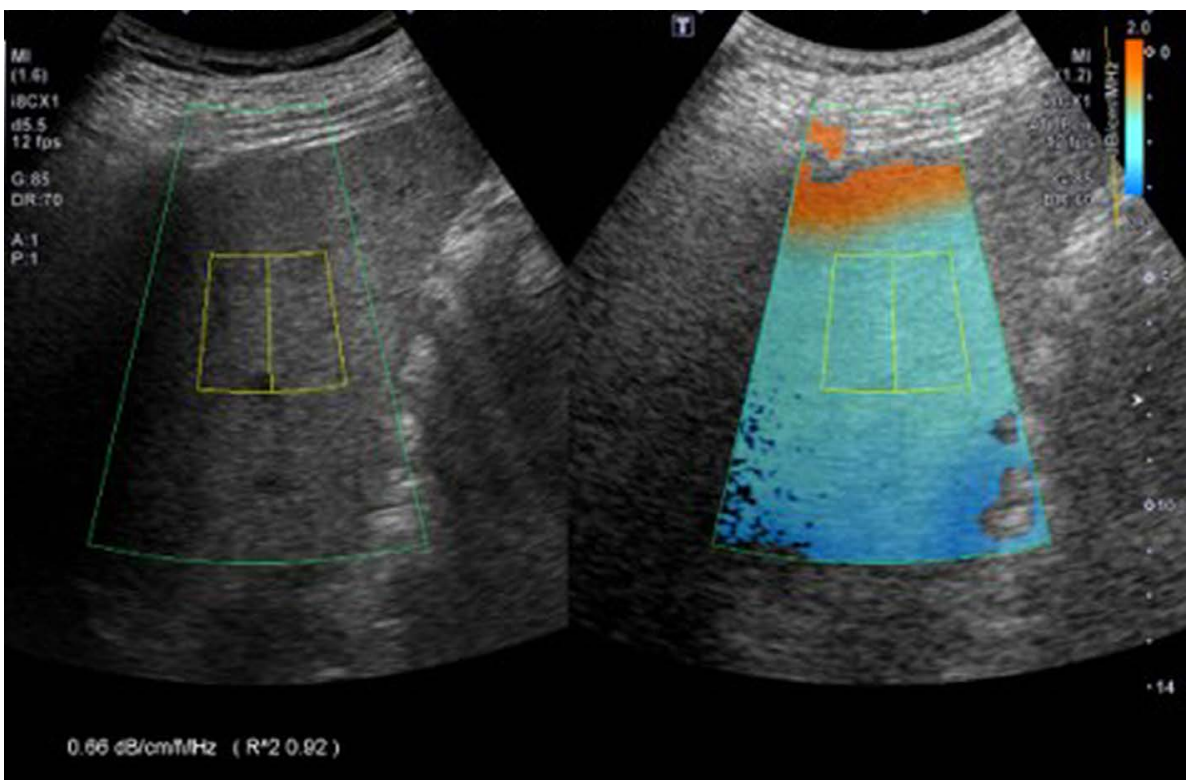

$\mathrm{E}$

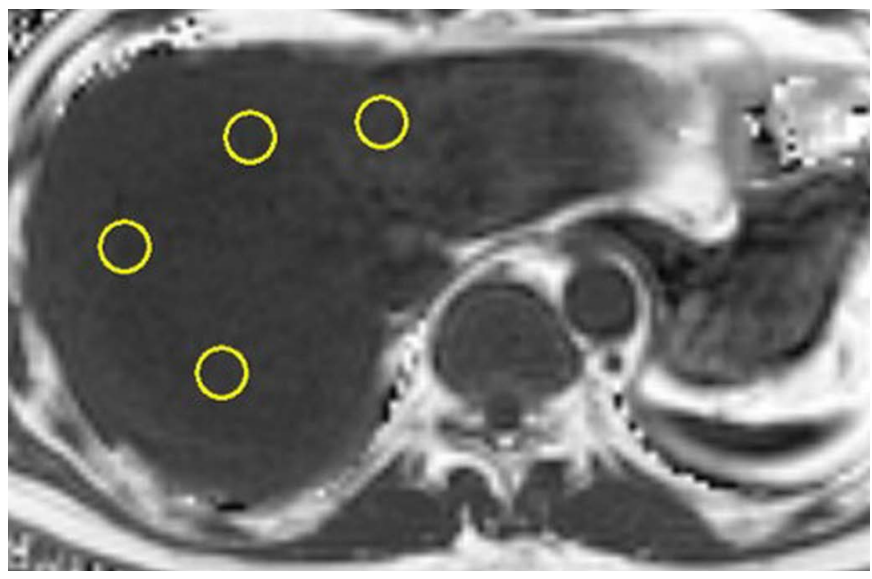

B

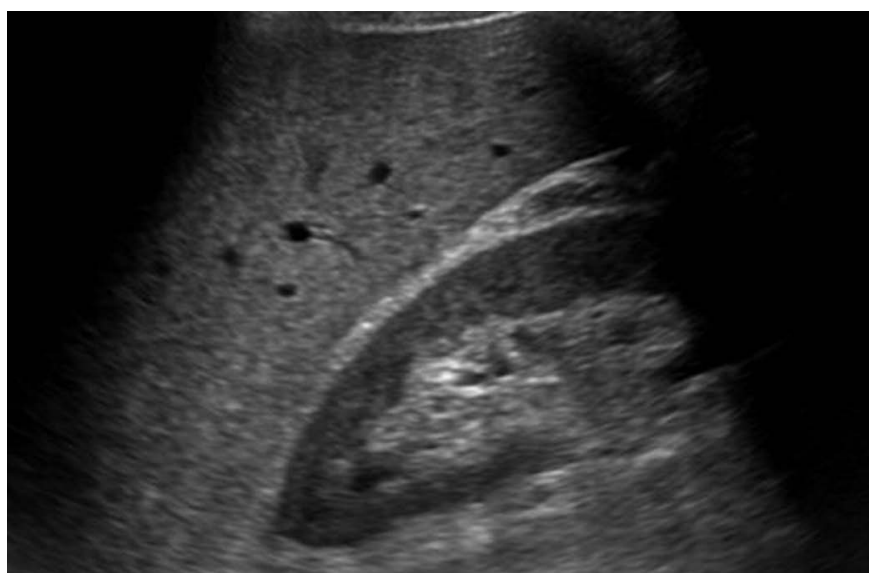

D

Fig. 2. Representative examples of the findings obtained using noninvasive tools in a 53-year-old man.

A. On unenhanced axial computed tomography images, $1.5 \times 1.5-\mathrm{cm}$ regions of interest (ROIs) (yellow squares) were placed in the liver and spleen. B. On a proton density fat fraction map of magnetic resonance imaging, $1 \times 1 \mathrm{~cm}$ ROls (yellow circles) were placed in each Couinaud segment of the liver. C. The controlled attenuation parameter was obtained from transient elastography. D. On grayscale ultrasound images, the echogenicity of the hepatic parenchyma was compared with that of the right kidney. E. Attenuation imaging was performed to measure the attenuation coefficient. On pathologic examination, the degree of hepatic steatosis was $\geq 5 \%-33 \%$. 
the ATI algorithm. An ROI was then placed in the middle portion of the sampling box avoiding the liver capsule for the measurement of the attenuation coefficient (AC) (dB/cm/MHz) (Fig. 2E). According to the manufacturer's recommendation, ATI examinations were performed until five valid $A C$ values were obtained. The median $A C$ value was used for the analysis. The details of the ATI examinations are provided in the Supplementary Data 1.

\section{Pathologic Examination}

Hepatic resection was performed in all patients, and histological slides stained using hematoxylin-eosin and Masson trichrome were prepared from surgical specimens. All surgical specimens were assessed by one pathologist (H.K., with 20 years of experience evaluating liver pathology) who was blinded to the results of the HS assessed based on the imaging examinations. The degree of HS was scored based on the percentage of macrovesicular lipid vacuolecontaining hepatocytes according to the NAFLD scoring system: $<5 \%, \geq 5 \%-33 \%$, $>33 \%-66 \%$, and $>66 \%$ [28]. HS of $\geq 5 \%-33 \%$ was further divided into two categories using a cutoff of $10 \%$, which is considered a safe threshold for liver donation [29]. In addition to the degree of HS, the degree of fibrosis and inflammatory activity were evaluated using the standardized guidelines proposed by the Korean Study Group for the Pathology of Digestive Diseases [30].

\section{Statistical Analysis}

Data are presented as the mean \pm standard deviation, median (interquartile range), or number (percentage), as appropriate. Continuous variables were compared using the independent t-test or the Mann-Whitney $U$ test, and categorical variables were compared using the chi-square test or the Fisher exact test. For comparisons between multiple groups, analysis of variance or Kruskal-Wallis test with the post-hoc Bonferroni correction was used. The diagnostic performance of each imaging tool in detecting varying degrees of HS was compared using the area under the curve (AUC) obtained from receiver operating characteristic curve analysis. Sensitivity, specificity, and positive and negative predictive values (PPVs and NPVs, respectively) were calculated by using the cutoff values obtained from the Youden index. Thereafter, the sensitivities and specificities of the imaging tools were compared using the McNemar test. A subgroup analysis was also performed in patients with HS $\geq 5 \%$. Lastly, an additional review of grayscale US, CT, and MRI-PDFF images for evaluating $\mathrm{HS}$ was performed by a second radiologist (D.H.L.) to assess interobserver agreement using intraclass correlation coefficients; ATI and CAP were not assessed owing to the retrospective nature of this study. All statistical analyses were performed using commercial statistical software (MedCalc, version 19.0.7, MedCalc Software, Ostend, Belgium). A two-sided P-value less than 0.05 was considered to indicate statistical significance.

\section{Results}

\section{Patients}

The baseline characteristics of the study participants are presented in Table 1. The median body mass index was $24.0 \mathrm{~kg} / \mathrm{m}^{2}$ (interquartile range, 22.5 to $\left.26.5 \mathrm{~kg} / \mathrm{m}^{2}\right)$. The majority of the patients had an underlying chronic liver disease due to viral infection $(66.7 \%$ [80/120]), and eight patients fulfilled the criteria for the diagnosis of NAFLD (i.e., without any primary chronic liver disease). Among the eight patients with NAFLD, two patients were diagnosed with NASH on pathology. The pathologic diagnosis of the tumors is presented in Supplementary Table 1. In terms of the degree of HS on pathology, there were 54 patients without HS (HS $<5 \%$ ), 50 patients with HS of $\geq 5 \%-33 \%, 14$ patients with HS of $>33 \%-66 \%$, and two patients with $\mathrm{HS}>66 \%$.

\section{Quantitative Estimation of Hepatic Steatosis}

The distribution of the quantitative estimates of HS on CT, MRI-PDFF, CAP, and ATI according to the degree of HS on pathology are shown in Fig. 3. The CT index decreased as the degree of HS increased, while the fat fraction measured by MRI-PDFF, CAP, and AC from ATI increased as the degree of HS increased. Except for HS $>66 \%(n=2)$, there was a significant difference between each group of different degrees of $\mathrm{HS}$ for the indices of all four quantitative imaging tools $(\mathrm{P}<0.01$ for all). The $\mathrm{P}$-values for each comparison are presented in Supplementary Table 2. The quantitative estimates of HS on CT, MRI-PDFF, CAP, and ATI did not differ significantly between groups according to different degrees of fibrosis on pathology ( $P>0.05$ for all) (Supplementary Fig. 1). For inflammatory activity, however, there was a significant difference between each group for the quantitative estimates of HS on CT and ATI ( $P=0.026$ and $P=0.045$, respectively) while there was no significant difference for MRI-PDFF and CAP $(P=0.104$ and $P=0.577$, respectively) (Supplementary Fig. 2). The post-hoc test revealed that with $\mathrm{CT}$, the estimates of HS of patients with moderate inflammatory activity $(n=7)$ were significantly lower than the values of patients with minimal activity $(n=63)$ $(P=0.006)$. However, the post-hoc test demonstrated that there was no significant difference using ATI between patients with different degrees of inflammatory activity ( $P \geq 0.012$, which is greater than the 0.008 threshold obtained by considering multiple comparisons). In terms of interobserver agreement, the intraclass correlation coefficients for grayscale US, CT, and MRI-PDFF were 0.68 (95\% confidence interval $[\mathrm{Cl}], 0.47$ to 0.80$), 0.95(95 \% \mathrm{Cl}, 0.87$ to 0.98$)$, and $0.97(95 \% \mathrm{Cl}, 0.93$ to 0.99$)$, respectively. 

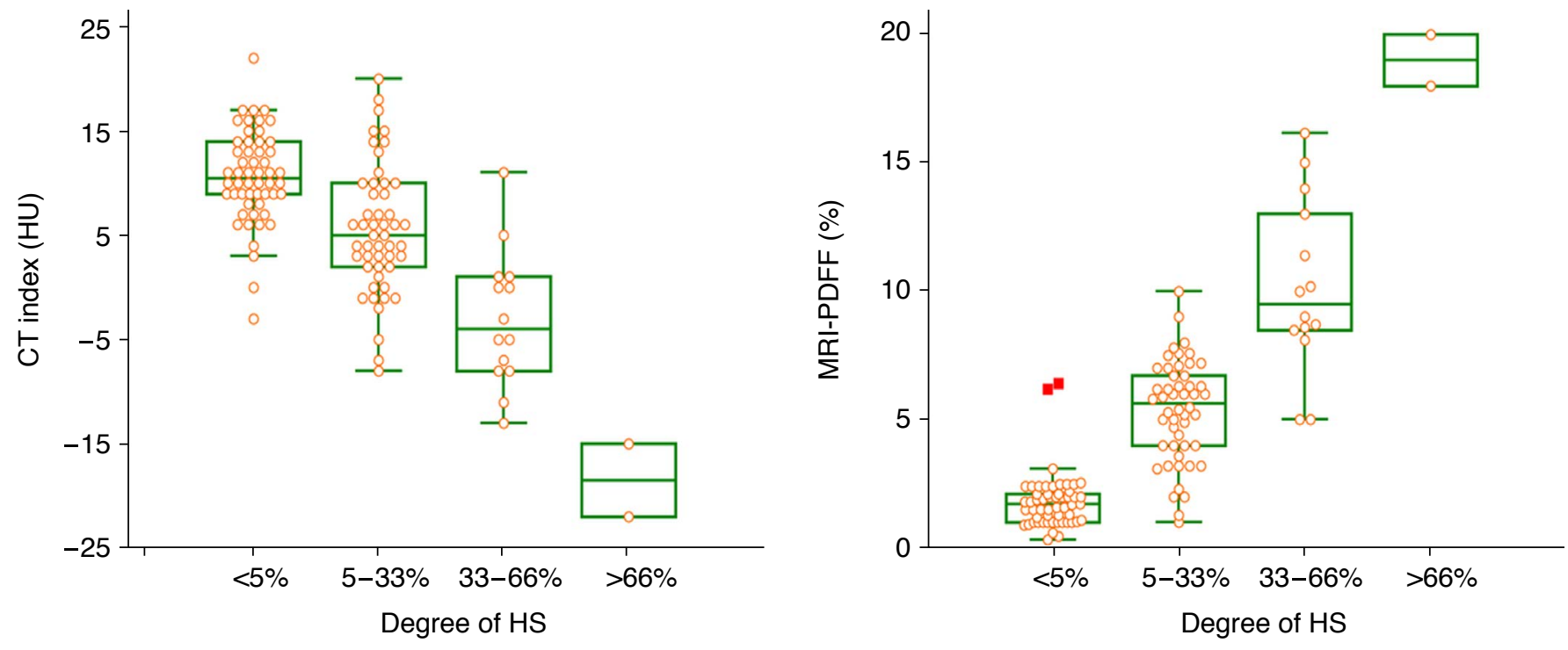

A

B
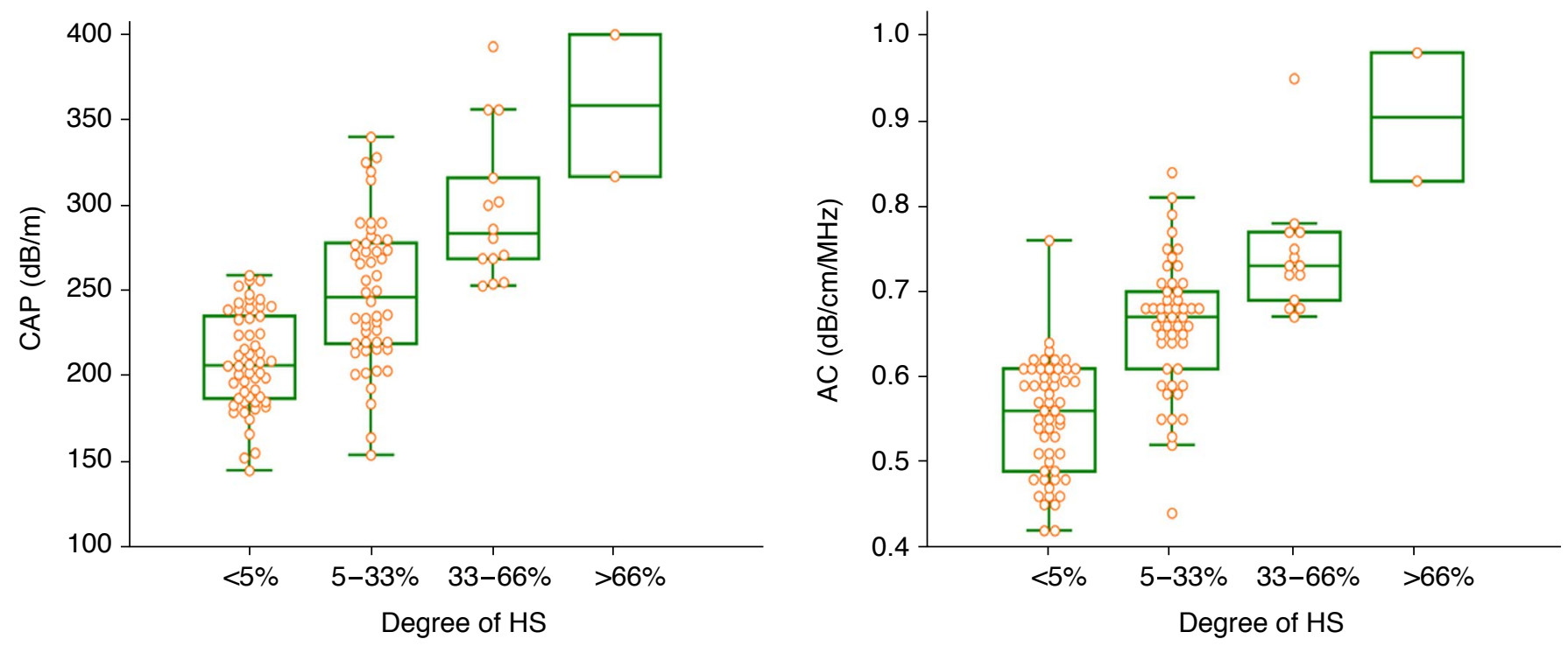

Fig. 3. The distribution of quantitative estimates obtained from CT (A), MRI-PDFF (B), CAP (C), and AC from attenuation imaging (D) according to the $\mathrm{HS}$ grade on pathology.

Red squares indicate outliers; central box represents the values from the lower to upper quartile ( 25 to 75 percentile); error bars indicate the $95 \%$ confidence intervals of the proportions. CT, computed tomography; HU, Hounsfield unit; HS, hepatic steatosis; MRI-PDFF, magnetic resonance imaging-derived proton density fat fraction; $\mathrm{CAP}$, controlled attenuation parameter; $\mathrm{AC}$, attenuation coefficient.

Comparison of Imaging Tools for Detecting Hepatic Steatosis For the detection of HS $\geq 5 \%$, the AUC of MRI-PDFF $(0.946 ; 95 \%$ $\mathrm{Cl}, 0.889$ to 0.979$)$ was significantly higher than that of $\mathrm{CT}(0.807$; $95 \% \mathrm{Cl}, 0.724$ to 0.873$)$, CAP $(0.829 ; 95 \% \mathrm{Cl}, 0.750$ to 0.892$)$, and grayscale US $(0.761 ; 95 \% \mathrm{Cl}, 0.674$ to 0.834$)(\mathrm{P}<0.01$ for all) (Table 2). In pairwise comparisons, there were no significant differences between the AUC of ATI and the values of MRI-PDFF, $C T$, or CAP $(P=0.133, P=0.063$, and $P=0.150$, respectively).
Meanwhile, the AUC of ATI was significantly higher than that of grayscale US ( $P=0.001$ ) (Fig. 4). For the detection of HS $>33 \%$, there was no significant difference in diagnostic performance among the various imaging tools ( $P>0.05$ for all) (Table 3, Fig. 4). The results for the detection of $\mathrm{HS} \geq 10 \%$ and for the subgroup analysis of patients with $\mathrm{HS} \geq 5 \%$ are summarized in Supplementary Table 3 and Supplementary Fig. 3, and Supplementary Table 4 and Supplementary Fig. 4, respectively. 
Table 2. Diagnostic performance of imaging tools for detecting $\mathrm{HS} \geq 5 \%$

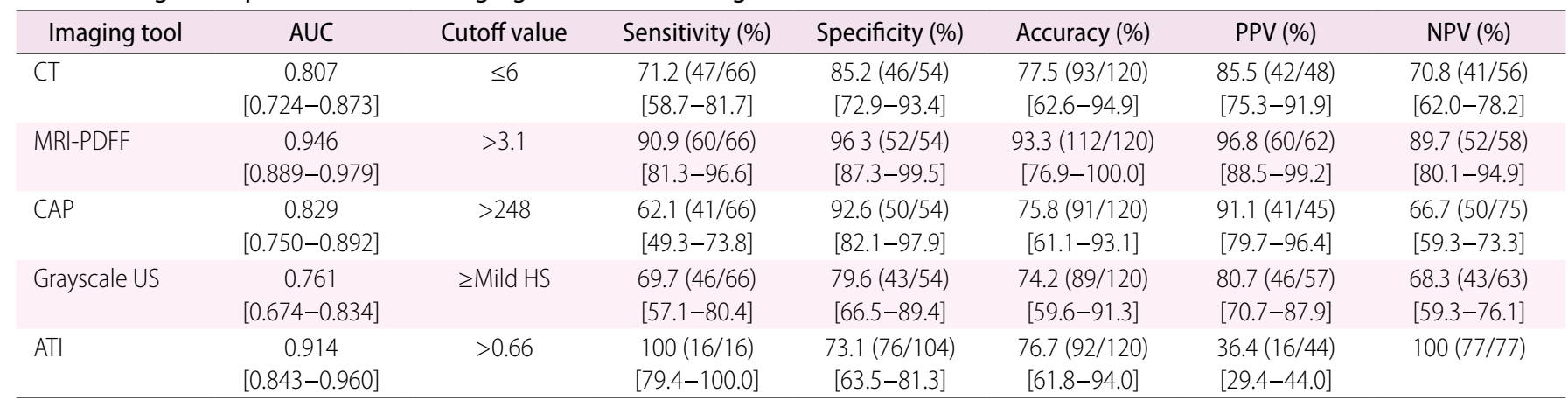

Values are percentages, with numerators and denominators in parentheses and $95 \%$ confidence intervals in brackets.

HS, hepatic steatosis; AUC, area under the curve; PPV, positive predictive value; NPV, negative predictive value; CT, computed tomography; MRI-PDFF, magnetic resonance imaging-derived proton density fat fraction; CAP, controlled attenuation parameter; US, ultrasound; ATI, attenuation imaging.

Table 3. Diagnostic performance of imaging tools for detecting $\mathrm{HS}>33 \%$

\begin{tabular}{|c|c|c|c|c|c|c|c|}
\hline Imaging tool & AUC & Cutoff value & Sensitivity (\%) & Specificity (\%) & Accuracy (\%) & PPV (\%) & NPV (\%) \\
\hline $\mathrm{CT}$ & $\begin{array}{c}0.887 \\
{[0.810-0.941]}\end{array}$ & $\leq 1$ & $\begin{array}{l}87.5(14 / 16) \\
{[61.7-98.4]}\end{array}$ & $\begin{array}{c}87.5(91 / 104) \\
{[79.6-93.2]}\end{array}$ & $\begin{array}{c}87.5(105 / 120) \\
{[71.6-100.0]}\end{array}$ & $\begin{array}{l}51.9(14 / 27) \\
{[38.5-64.9]}\end{array}$ & $\begin{array}{l}97.8(91 / 93) \\
{[92.5-99.4]}\end{array}$ \\
\hline MRI-PDFF & $\begin{array}{c}0.947 \\
{[0.884-0.981]}\end{array}$ & $>8$ & $\begin{array}{l}87.5(14 / 16) \\
{[61.7-98.4]}\end{array}$ & $\begin{array}{c}98.1(102 / 104) \\
{[93.2-99.8]}\end{array}$ & $\begin{array}{c}96.7(116 / 120) \\
{[79.9-100.0]}\end{array}$ & $\begin{array}{l}87.5(14 / 16) \\
{[63.7-96.5]}\end{array}$ & $\begin{array}{c}98.1(102 / 104) \\
{[93.3-99.5]}\end{array}$ \\
\hline CAP & $\begin{array}{c}0.900 \\
{[0.826-0.950]}\end{array}$ & $>250$ & $\begin{array}{c}100(16 / 16) \\
{[79.4-100.0]}\end{array}$ & $\begin{array}{c}74.0(77 / 104) \\
{[64.5-82.1]}\end{array}$ & $\begin{array}{c}77.5(93 / 120) \\
{[62.6-94.9]}\end{array}$ & $\begin{array}{l}37.2(16 / 43) \\
{[30.0-45.0]}\end{array}$ & $100(77 / 77)$ \\
\hline Grayscale US & $\begin{array}{c}0.914 \\
{[0.842-0.960]}\end{array}$ & $\geq$ Moderate HS & $\begin{array}{l}62.5(10 / 16) \\
{[35.4-84.8]}\end{array}$ & $\begin{array}{c}96.2(100 / 104) \\
{[90.4-98.9]}\end{array}$ & $\begin{array}{c}91.7(110 / 120) \\
{[75.3-100.0]}\end{array}$ & $\begin{array}{l}71.4(10 / 14) \\
{[47.1-87.5]}\end{array}$ & $\begin{array}{c}94.3(100 / 106) \\
{[89.8-96.9]}\end{array}$ \\
\hline ATI & $\begin{array}{c}0.914 \\
{[0.843-0.960]}\end{array}$ & $>0.66$ & $\begin{array}{c}100(16 / 16) \\
{[79.4-100.0]}\end{array}$ & $\begin{array}{c}73.1(76 / 104) \\
{[63.5-81.3]}\end{array}$ & $\begin{array}{c}76.7(92 / 120) \\
{[61.8-94.0]}\end{array}$ & $\begin{array}{l}36.4(16 / 44) \\
{[29.4-44.0]}\end{array}$ & $100(77 / 77)$ \\
\hline
\end{tabular}

Values are percentages, with numerators and denominators in parentheses and $95 \%$ confidence intervals in brackets.

HS, hepatic steatosis; AUC, area under the curve; PPV, positive predictive value; NPV, negative predictive value; CT, computed tomography; MRI-PDFF, magnetic resonance imaging-derived proton density fat fraction; CAP, controlled attenuation parameter; US, ultrasound; ATI, attenuation imaging.

The cutoff value, sensitivity, specificity, accuracy, PPV, and NPV of the imaging tools for detecting $\mathrm{HS} \geq 5 \%$ and $\mathrm{HS}>33 \%$ are presented in Tables 2 and 3, respectively. For detecting HS $\geq 5 \%$, MRI-PDFF showed the highest sensitivity (90.9\%), and CAP showed the lowest sensitivity (62.1\%). Meanwhile, MRI-PDFF and ATI showed the highest specificity (96.3\%), and grayscale US showed the lowest specificity (79.6\%). For detecting $\mathrm{HS}>33 \%$, grayscale US showed significantly lower sensitivity (62.5\%) than CAP and ATI (100\% for both) ( $P=0.03$ for both). Regarding specificity, MRI-PDFF demonstrated the highest value (98.1\%), which was significantly higher than $C T, C A P$, and ATI ( $P<0.001$ for all). The detailed results of the pairwise comparisons among the imaging tools for sensitivity and specificity for detecting $\mathrm{HS} \geq 5 \%$ and $\mathrm{HS}>33 \%$ are presented in Supplementary Tables 5 and 6 . The results of the pairwise comparisons for detecting $\mathrm{HS} \geq 10 \%$ are presented in Supplementary Table 7.

\section{Discussion}

The present study demonstrated that MRI-PDFF significantly outperformed CT, CAP, and grayscale US (AUC, 0.946 vs. $0.761-$ $0.829)(P<0.01$ for all) but was not significantly better than ATI (AUC, 0.892; $\mathrm{P}=0.133$ ) in the detection of $\mathrm{HS}$. For detecting $\mathrm{HS}>33 \%$, all of the imaging tools demonstrated comparable, satisfactory diagnostic performances (AUC, 0.887 to 0.947), and MRI-PDFF had the highest accuracy (96.7\%). This result suggests that MRIPDFF is the most accurate imaging modality in detecting patients with $H S$, which reconfirms the results of previous studies $[20,31]$. In particular, considering that patients with metabolic syndrome or NASH commonly have only a mild degree of HS [32], MRI-PDFF may be the best imaging modality for screening patients for $\mathrm{HS}$. However, MRI-PDFF has drawbacks such as high cost and limited availability, which limits its use as a screening tool [7]. Meanwhile, ATI significantly outperformed grayscale US in detecting $\mathrm{HS} \geq 5 \%$. As a two-dimensional US image-based technique, ATI retains the 


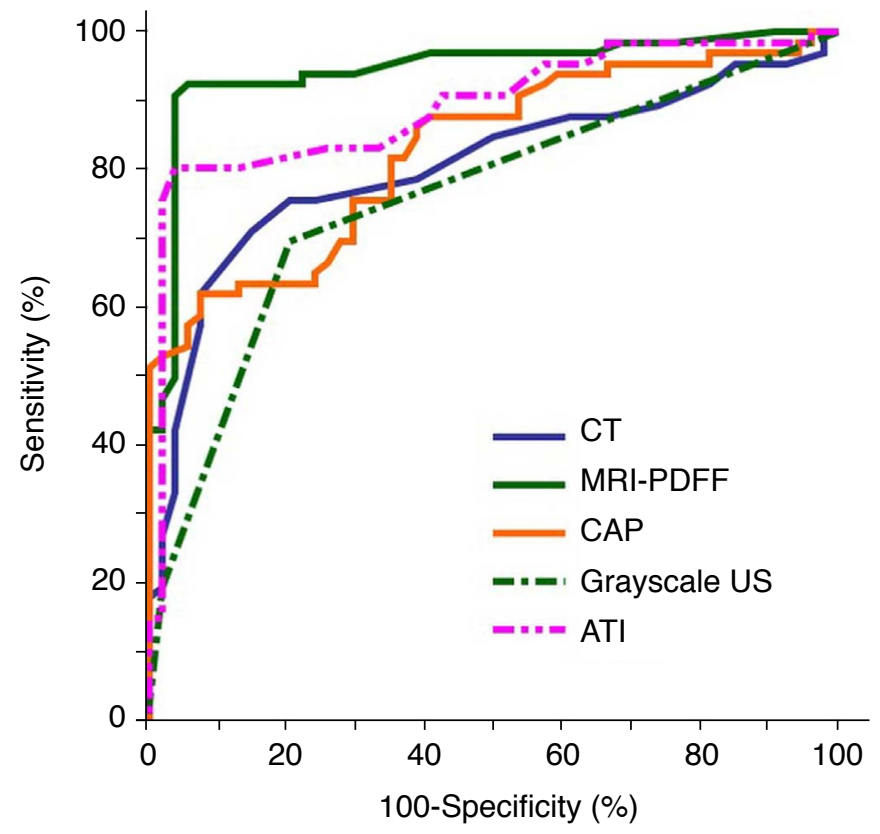

A

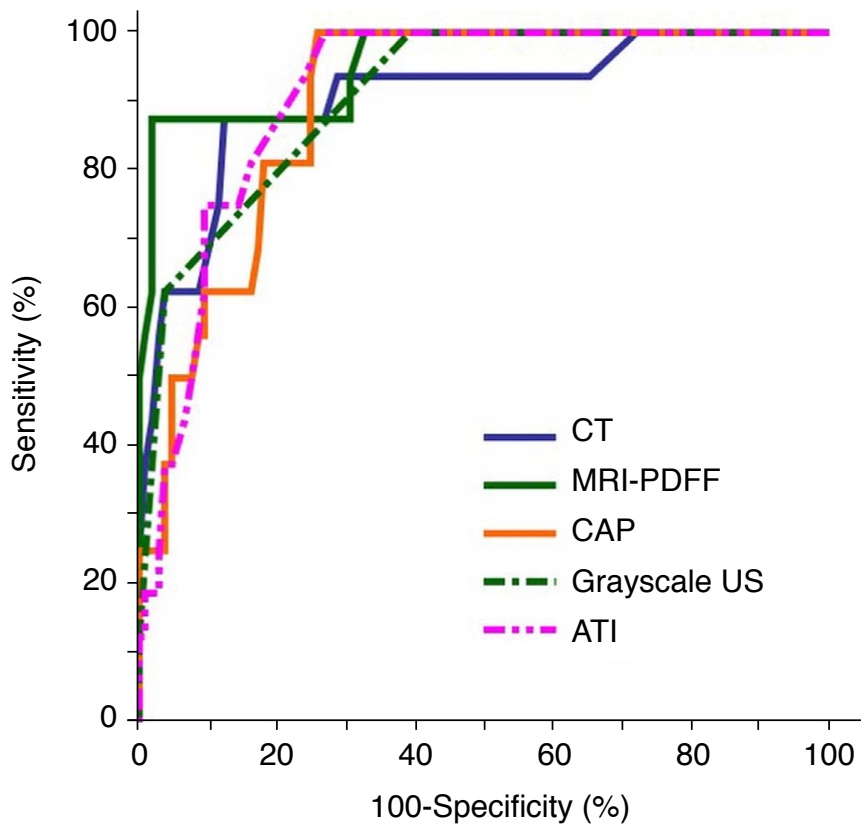

B

Fig. 4. Comparison of receiver operating characteristic curves among the five imaging tools for detecting $\geq 5 \%$ (A) and $>33 \%$ (B) hepatic steatosis on pathology.

CT, computed tomography; MRI-PDFF, magnetic resonance imaging-derived proton density fat fraction; CAP, controlled attenuation parameter; US, ultrasound; ATI, attenuation imaging.

advantage of US, including high accessibility, relatively low cost, and no radiation exposure [8], although it is a vendor-specific technique. Compared with CAP, ATI can easily be performed during a grayscale US examination, obviating the need to perform a separate examination (i.e., TE). Moreover, the evaluation of hepatic parenchyma for both the degree of $\mathrm{HS}$ and the presence of focal liver lesions is possible with ATI, but not with CAP. Therefore, ATI may be more suitable than grayscale US or CAP as a screening tool to assess patients for HS. If patients are positive on ATI, MRI-PDFF could be used as a confirmatory tool, considering its high accuracy but limited availability. The present study may have essential clinical value for clinicians who diagnose and treat patients with $\mathrm{HS}$, as the present study compared the diagnostic performance of representative noninvasive tools for the evaluation of HS using pathologic results as a reference standard.

Although both ATI and CAP share the same physics of attenuation of US [9,33], ATI is based on two-dimensional images that can be used to evaluate the hepatic parenchyma while avoiding large vessels or focal liver lesions, whereas CAP lacks two-dimensional images. Therefore, CAP has a theoretical disadvantage for evaluating HS compared to ATI, especially in patients with focal liver lesions in the right lobe of the liver where CAP is measured. In addition, the sample volume of ATI from grayscale US images is larger than that of CAP $[9,34]$. The lower diagnostic performance of CAP than
ATI in detecting $\mathrm{HS} \geq 5 \%$ in this study might have been caused by this inherent limitation of CAP, although the difference was not statistically significant. A similar tendency was observed in some previous studies that compared ATI and CAP for detecting HS $[13,15]$. However, CAP has been extensively validated in various clinical scenarios, and the impact of technical details on its accuracy, including the selection of probes tailored to patients, has been established $[34,35]$, while ATI has only been recently introduced into clinical practice and requires further validation. Therefore, further studies comparing ATI to other quantitative tools, including CAP, in different clinical scenarios (e.g., patients with or without focal liver lesions) are warranted to more accurately establish the role of ATI in the management of fatty liver disease.

Although detecting the presence of HS itself is required to diagnose patients with NAFLD, ruling in/out patients with moderate or severe HS (i.e., HS $>33 \%$ ) has clinical importance, as it is associated with NASH and progression to cirrhosis and liver cancer in patients with NAFLD [36]. Moderate or severe HS has also been reported to be associated with complications following hepatic resection and the coronary artery atherosclerosis burden $[37,38]$. In the present study, all five imaging modalities provided satisfactory, comparable diagnostic performance for the detection of $\mathrm{HS}>33 \%$ in terms of the AUC (0.887-0.947). However, a closer look into the results reveals that MRI-PDFF demonstrated the highest specificity 
(98.1\%), while CAP and ATI, which are both attenuation-based examinations, showed the highest sensitivity values $(100 \%$ for both). Indeed, the AUC of MRI-PDFF to detect HS $>33 \%$ was higher than that of ATI or CAP, although a statistically significant difference was not achieved. Considering these results, the authors speculate that either CAP or ATI might be used as a screening tool for the detection of patients with $\mathrm{HS}>33 \%$, and MRI-PDFF, with its high specificity, could be used as a confirmatory noninvasive diagnostic method for patients with positive results on ATI or CAP.

There are a few limitations of the present study that should be noted. First, the study participants consisted of relatively lean surgical candidates, mainly with chronic viral hepatitis infections. In the general population or among patients with NAFLD with a higher degree of HS than the surgical candidates in this study, the diagnostic performance of the imaging tools may be different from the present results. Second, the degree of HS on pathology was categorized but not quantitatively estimated. Thus, the correlation between the exact amount of HS on pathology and the results of quantitative imaging tools was not assessed. Third, the degree of iron deposition in the liver was not assessed on pathology. However, the MRI-PDFF technique has a $\mathrm{T}^{*}$ correction that could compensate for the effect of iron, and the prevalence of hereditary hemochromatosis or $\beta$-thalassemia, which could result in hepatic iron overload, is very low in the East Asian population [39]. Thus, in the authors' opinion, the effect of iron overload in the evaluation of HS may not have been significant. Fourth, the CT index may have been affected by using peak tube voltages of both $100 \mathrm{kVp}$ and $120 \mathrm{kVp}$ [40], although most examinations (92.5\% [111/120]) were obtained with $100 \mathrm{kVp}$. In addition, the degree of HS may have changed in the time interval of 3 months between the imaging study and surgery. Lastly, the interobserver agreement for ATI and CAP was not assessed due to the retrospective nature of this study.

In conclusion, MRI-PDFF was the best imaging modality for detecting any degree of $\mathrm{HS}$ based on pathologic reference standards, while ATI outperformed grayscale US. In detecting HS $>33 \%$, all five imaging tools demonstrated good diagnostic performance.

ORCID: Jae Seok Bae: https://orcid.org/0000-0003-2768-7917; Dong Ho Lee: https:// orcid.org/0000-0001-8983-851X; Kyung-Suk Suh: https://orcid.org/0000-0002-95357349; Haeryoung Kim: https://orcid.org/0000-0002-4205-9081; Kyung Bun Lee: https:// orcid.org/0000-0001-8427-3003; Jae Young Lee: https://orcid.org/0000-0001-69466042; Joon Koo Han: https://orcid.org/0000-0001-5916-5545

\section{Author Contributions}

Conceptualization: Bae JS, Lee DH, Lee JY, Han JK. Data acquisition: Bae JS, Lee DH, Suh KS, Lee KB, Kim H. Data analysis or interpretation: Bae JS, Lee DH. Drafting of the manuscript: Bae JS,
Lee DH. Critical revision of the manuscript: Bae JS, Lee DH, Suh KS, Lee KB, Kim H, Lee JY, Han JK. Approval of the final version of the manuscript: all authors.

\section{Conflict of Interest}

No potential conflict of interest relevant to this article was reported.

\section{Supplementary Material}

Supplementary Data 1. Details of imaging examinations (https:// doi.org/10.14366/usg.21150).

Supplementary Table 1. Pathologic diagnosis of tumor (https:// doi.org/10.14366/usg.21150).

Supplementary Table 2. Comparison between each group of different degrees of hepatic steatosis by using quantitative imaging tools (https://doi.org/10.14366/usg.21150).

Supplementary Table 3. Diagnostic performance of imaging tools for detecting HS $\geq 10 \%$ (https://doi.org/10.14366/usg.21150).

Supplementary Table 4. Subgroup analysis: Diagnostic performance of imaging tools for detecting $\mathrm{HS}>33 \%$ in patients with $\mathrm{HS} \geq 5 \%$ (https://doi.org/10.14366/usg.21150).

Supplementary Table 5. Difference in sensitivity and specificity between imaging tools for detecting hepatic steatosis $\geq 5 \%$ (https:// doi.org/10.14366/usg.21150).

Supplementary Table 6. Difference in sensitivity and specificity between imaging tools for detecting hepatic steatosis $>33 \%$ (https:// doi.org/10.14366/usg.21150).

Supplementary Table 7. Difference in sensitivity and specificity between imaging tools for detecting hepatic steatosis $\geq 10 \%$ (https:// doi.org/10.14366/usg.21150).

Supplementary Fig. 1. The distribution of quantitative estimates obtained from $C T(A)$, MRI-PDFF (B), CAP (C), and AC from attenuation imaging $(D)$ according to the hepatic fibrosis grade on pathology. Red squares indicate outliers; central box represents the values from the lower to upper quartile ( 25 to 75 percentile); error bars indicate the $95 \%$ confidence intervals of the proportions. CT, computed tomography; HU, Hounsfield unit; MRI-PDFF, magnetic resonance imaging-derived proton density fat fraction; $C A P$, controlled attenuation parameter; $A C$, attenuation coefficient (https://doi.org/10.14366/usg.21150). 
Supplementary Fig. 2. The distribution of quantitative estimates obtained from CT (A), MRI-PDFF (B), CAP (C), and AC from attenuation imaging (D) according to the grade of hepatic inflammatory activity on pathology. Central box represents the values from the lower to upper quartile ( 25 to 75 percentile); error bars indicate the $95 \%$ confidence intervals of the proportions. CT, computed tomography; HU, Hounsfield unit; MRI-PDFF, magnetic resonance imaging-derived proton density fat fraction; $C A P$, controlled attenuation parameter; $A C$, attenuation coefficient (https://doi.org/10.14366/usg.21150).

Supplementary Fig. 3. Comparison of receiver operator characteristic curves among the five imaging tools for detecting $\geq 10 \%$ hepatic steatosis on pathology. CT, computed tomography; MRI-PDFF, magnetic resonance imaging-derived proton density fat fraction; CAP, controlled attenuation parameter; US, ultrasonography; ATI, attenuation imaging (https://doi.org/10.14366/usg.21150).

Supplementary Fig. 4. Subgroup analysis: comparison of receiver operator characteristic curves among the five imaging tools for detecting $\geq 33 \%$ hepatic steatosis on pathology in patients with hepatic steatosis $\geq 5 \%$. CT, computed tomography; MRI-PDFF, magnetic resonance imaging-derived proton density fat fraction; CAP, controlled attenuation parameter; US, ultrasonography; ATI, attenuation imaging (https://doi.org/10.14366/usg.21150).

\section{References}

1. Ress C, Kaser S. Mechanisms of intrahepatic triglyceride accumulation. World J Gastroenterol 2016;22:1664-1673.

2. Paik JM, Golabi P, Younossi Y, Mishra A, Younossi ZM. Changes in the global burden of chronic liver diseases from 2012 to 2017: the growing impact of NAFLD. Hepatology 2020;72:1605-1616.

3. Noureddin M, Vipani A, Bresee C, Todo T, Kim IK, Alkhouri N, et al. NASH leading cause of liver transplant in women: updated analysis of indications for liver transplant and ethnic and gender variances. Am J Gastroenterol 2018;113:1649-1659.

4. Sheka AC, Adeyi O, Thompson J, Hameed B, Crawford PA, Ikramuddin S. Nonalcoholic steatohepatitis: a review. JAMA 2020;323:1175-1183.

5. Kessoku T, Imajo K, Kobayashi T, Ozaki A, Iwaki M, Honda Y, et al. Lubiprostone in patients with non-alcoholic fatty liver disease: a randomised, double-blind, placebo-controlled, phase 2a trial. Lancet Gastroenterol Hepatol 2020;5:996-1007.

6. Machado MV, Cortez-Pinto H. Non-invasive diagnosis of nonalcoholic fatty liver disease: a critical appraisal. J Hepatol 2013;58:1007-1019.
7. Lee DH. Imaging evaluation of non-alcoholic fatty liver disease: focused on quantification. Clin Mol Hepatol 2017;23:290-301.

8. Ferraioli G, Soares Monteiro LB. Ultrasound-based techniques for the diagnosis of liver steatosis. World J Gastroenterol 2019;25:6053-6062.

9. Bae JS, Lee DH, Lee JY, Kim H, Yu SJ, Lee JH, et al. Assessment of hepatic steatosis by using attenuation imaging: a quantitative, easy-to-perform ultrasound technique. Eur Radiol 2019;29:64996507.

10. Lee DH, Cho EJ, Bae JS, Lee JY, Yu SJ, Kim H, et al. Accuracy of twodimensional shear wave elastography and attenuation imaging for evaluation of patients with nonalcoholic steatohepatitis. Clin Gastroenterol Hepatol 2021;19:797-805.

11. Yoo J, Lee JM, Joo I, Lee DH, Yoon JH, Kang HJ, et al. Reproducibility of ultrasound attenuation imaging for the noninvasive evaluation of hepatic steatosis. Ultrasonography 2020;39:121-129.

12. Jeon SK, Lee JM, Joo I, Yoon JH, Lee DH, Lee JY, et al. Prospective evaluation of hepatic steatosis using ultrasound attenuation imaging in patients with chronic liver disease with magnetic resonance imaging proton density fat fraction as the reference standard. Ultrasound Med Biol 2019;45:1407-1416.

13. Ferraioli G, Maiocchi L, Raciti MV, Tinelli C, De Silvestri A, Nichetti $M$, et al. Detection of liver steatosis with a novel ultrasoundbased technique: a pilot study using MRI-derived proton density fat fraction as the gold standard. Clin Transl Gastroenterol 2019;10:e00081.

14. Dioguardi Burgio M, Ronot M, Reizine E, Rautou PE, Castera L, Paradis $V$, et al. Quantification of hepatic steatosis with ultrasound: promising role of attenuation imaging coefficient in a biopsyproven cohort. Eur Radiol 2020;30:2293-2301.

15. Ferraioli G, Maiocchi L, Savietto G, Tinelli C, Nichetti M, Rondanelli $M$, et al. Performance of the attenuation imaging technology in the detection of liver steatosis. J Ultrasound Med 2021;40:1325-1332.

16. Tada T, lijima H, Kobayashi $N$, Yoshida M, Nishimura T, Kumada T, et al. Usefulness of attenuation imaging with an ultrasound scanner for the evaluation of hepatic steatosis. Ultrasound Med Biol 2019;45:2679-2687.

17. Sugimoto K, Moriyasu F, Oshiro H, Takeuchi H, Abe M, Yoshimasu Y, et al. The role of multiparametric US of the liver for the evaluation of nonalcoholic steatohepatitis. Radiology 2020;296:532-540.

18. Lee SS, Park SH, Kim HJ, Kim SY, Kim MY, Kim DY, et al. Noninvasive assessment of hepatic steatosis: prospective comparison of the accuracy of imaging examinations. J Hepatol 2010;52:579-585.

19. van Werven JR, Marsman HA, Nederveen AJ, Smits NJ, ten Kate FJ, van Gulik TM, et al. Assessment of hepatic steatosis in patients undergoing liver resection: comparison of US, CT, T1-weighted dual-echo MR imaging, and point-resolved $1 \mathrm{H}$ MR spectroscopy. Radiology 2010;256:159-168.

20. Kramer H, Pickhardt PJ, Kliewer MA, Hernando D, Chen GH, 
Zagzebski JA, et al. Accuracy of liver fat quantification with advanced $C T, M R I$, and ultrasound techniques: prospective comparison with MR spectroscopy. AJR Am J Roentgenol 2017;208:92-100.

21. Eddowes PJ, Sasso M, Allison M, Tsochatzis E, Anstee QM, Sheridan D, et al. Accuracy of FibroScan controlled attenuation parameter and liver stiffness measurement in assessing steatosis and fibrosis in patients with nonalcoholic fatty liver disease. Gastroenterology 2019;156:1717-1730.

22. Lee DH, Lee ES, Bae JS, Lee JY, Han JK, Yi NJ, et al. 2D shear wave elastography is better than transient elastography in predicting post-hepatectomy complication after resection. Eur Radiol 2021;31:5802-5811.

23. Park SH, Kim PN, Kim KW, Lee SW, Yoon SE, Park SW, et al. Macrovesicular hepatic steatosis in living liver donors: use of $\mathrm{CT}$ for quantitative and qualitative assessment. Radiology 2006;239:105112.

24. Yokoo T, Serai SD, Pirasteh A, Bashir MR, Hamilton G, Hernando $D$, et al. Linearity, bias, and precision of hepatic proton density fat fraction measurements by using MR imaging: a meta-analysis. Radiology 2018;286:486-498.

25. Pooler BD, Wiens CN, McMillan A, Artz NS, Schlein A, Covarrubias $\mathrm{Y}$, et al. Monitoring fatty liver disease with MRI following bariatric surgery: a prospective, dual-center study. Radiology 2019;290:682690.

26. Wong JS, Wong GL, Chan AW, Wong VW, Cheung YS, Chong CN, et al. Liver stiffness measurement by transient elastography as a predictor on posthepatectomy outcomes. Ann Surg 2013;257:922928.

27. Dasarathy S, Dasarathy J, Khiyami A, Joseph R, Lopez R, McCullough AJ. Validity of real time ultrasound in the diagnosis of hepatic steatosis: a prospective study. J Hepatol 2009;51:1061-1067.

28. Kleiner DE, Brunt EM, Van Natta M, Behling C, Contos MJ, Cummings $\mathrm{OW}$, et al. Design and validation of a histological scoring system for nonalcoholic fatty liver disease. Hepatology 2005;41:1313-1321.

29. Sharma A, Ashworth A, Behnke M, Cotterell A, Posner M, Fisher RA. Donor selection for adult-to-adult living donor liver transplantation: well begun is half done. Transplantation 2013;95:501-506.

30. Park YN, Kim H, Chon CY, Park JB, Sohn JH, Yang SH, et al. Histological grading and staging of chronic hepatitis: standardized guideline proposed by the Korean Study Group for the Pathology of Digestive Diseases. Korean J Pathol 1999;33:337-346.

31. Imajo K, Kessoku T, Honda Y, Tomeno W, Ogawa Y, Mawatari H, et al. Magnetic resonance imaging more accurately classifies steatosis and fibrosis in patients with nonalcoholic fatty liver disease than transient elastography. Gastroenterology 2016;150:626-637.

32. Gholam PM, Flancbaum L, Machan JT, Charney DA, Kotler DP. Nonalcoholic fatty liver disease in severely obese subjects. Am J Gastroenterol 2007;102:399-408.

33. Sasso $M$, Beaugrand $M$, de Ledinghen $V$, Douvin $C$, Marcellin P, Poupon R, et al. Controlled attenuation parameter (CAP): a novel VCTE guided ultrasonic attenuation measurement for the evaluation of hepatic steatosis: preliminary study and validation in a cohort of patients with chronic liver disease from various causes. Ultrasound Med Biol 2010;36:1825-1835.

34. de Ledinghen V, Vergniol J, Capdepont M, Chermak F, Hiriart JB, Cassinotto $\mathrm{C}$, et al. Controlled attenuation parameter (CAP) for the diagnosis of steatosis: a prospective study of 5323 examinations. J Hepatol 2014;60:1026-1031.

35. Wong VW, Petta S, Hiriart JB, Camma C, Wong GL, Marra F, et al. Validity criteria for the diagnosis of fatty liver by $\mathrm{M}$ probe-based controlled attenuation parameter. J Hepatol 2017;67:577-584.

36. Festi D, Colecchia A, Sacco T, Bondi M, Roda E, Marchesini G. Hepatic steatosis in obese patients: clinical aspects and prognostic significance. Obes Rev 2004;5:27-42.

37. Kooby DA, Fong Y, Suriawinata A, Gonen M, Allen PJ, Klimstra DS, et al. Impact of steatosis on perioperative outcome following hepatic resection. J Gastrointest Surg 2003;7:1034-1044.

38. Hsu PF, Wang YW, Lin CC, Wang YJ, Ding YZ, Liou TL, et al. The association of the steatosis severity in fatty liver disease with coronary plaque pattern in general population. Liver Int 2021;41:81-90.

39. Wallace DF, Subramaniam VN. The global prevalence of HFE and non-HFE hemochromatosis estimated from analysis of nextgeneration sequencing data. Genet Med 2016;18:618-626.

40. Byun J, Lee SS, Sung YS, Shin Y, Yun J, Kim HS, et al. CT indices for the diagnosis of hepatic steatosis using non-enhanced CT images: development and validation of diagnostic cut-off values in a large cohort with pathological reference standard. Eur Radiol 2019;29:4427-4435. 